\title{
Design of Multifunctional Intelligent Car Based on Bluetooth Remote Control
}

\author{
$\mathrm{Ru} \mathrm{Nie}^{1, \mathrm{a}}$ \\ ${ }^{1}$ College of Electronic information engineering, Guangzhou College of South China \\ University of Technology, Guangzhou \\ a52811055@qq.com
}

Keywords: Single chip microcomputer; Bluetooth remote control; PWM speed control; photoelectric sensor.

\begin{abstract}
Wireless remote control robot car could play a special role in the hazardous environment operations and search \& rescue personnel. A multi-function smart car with Bluetooth remote control is selected as my graduation thesis subject. The design of the system hardware circuit schematic and PCB diagram is present in paper. The control system circuit is mainly consist of a STC12C5A60S2 MCU as the main chip, as well as a L293D chip for motor driving, a Bluetooth wireless communication module for remote control, an infrared photoelectric sensor module for object detection, an ultrasonic transmitter and receiver module for distance measurement. The smart car is implemented by the combination of the self-control circuit, the control program code and four-wheel car mechanical structure. Experimental tests showed that some functions such as Bluetooth wireless remote control, automatic obstacle avoidance, automatic tracking route, auto-sensing objects and auto-detecting distance were completely achieved in the smart car.
\end{abstract}

\section{Introduction}

With the rapid development of the automobile industry, the research on automobile is more and more popular. Remote control car originated in the United States, due to the government's support for the development of wireless remote control car and related support to promote the role of Japan, the United States, Germany and other industrial powers in the remote control car technology has a clear advantage. Research work of the wireless remote control car in China began in the late twentieth Century, with the support of the National 863, 973 and other technical development plans, the domestic has been widely carried out research on wireless remote control car. National electronic contest and the province of electronic competition almost every time there are intelligent car, the National University also attach great importance to the subject of research, but with the international advanced there is a certain gap, it is very important to study the design is in such a background. Wireless remote control method including Bluetooth, infrared, radio frequency, which has a certain advantage of Bluetooth technology, the application of information appliances is being laid, a variety of home appliances shared remote control, and can be connected to the public Internet and public Internet, sharing useful information. And the Bluetooth technology to realize wireless remote control of short board is transmission distance is short and chip prices, with the development of science and technology, is gradually being resolved, Bluetooth wireless remote control has broad application prospects [1].

This design is based on the Bluetooth wireless remote control, the installation of photoelectric infrared sensor, ultrasonic sensor, photosensitive resistance, temperature sensor, the electric vehicle speed, position, operating condition, real-time monitoring, and the measurement data is transmitted to the single chip microcomputer. 


\section{System hardware design}

2.1 The overall design of intelligent car control system

This car is the STC series STC12C5A60S2 controller. First, the computer or mobile phone to send a Bluetooth wireless signal to start and reset the car, and then by ultrasonic sensor or infrared sensor for obstacle detection, control of the car through the microcontroller, display, avoid obstacles and speed. Smart car use 4WD drive, in order to improve the smoothness of the vehicle movement; in intelligent vehicle in the driving process, the bipolar type H-type PWM pulse width modulation technology quickly and steadily to achieve speed; through ultrasonic sensors and infrared photoelectric sensor to achieve automatic obstacle avoidance, automatic tracking and other functions; through the transmission type photoelectric sensor measuring wheel rotating circle number (which is the pulse number) to achieve speed detection function; finally through Bluetooth wireless transmission function will be intelligent vehicle driving information transmitted in real time to the host computer, in order to realize the real-time monitoring function. Of course, it can also be used to control the state of the car through the Bluetooth wireless remote control.

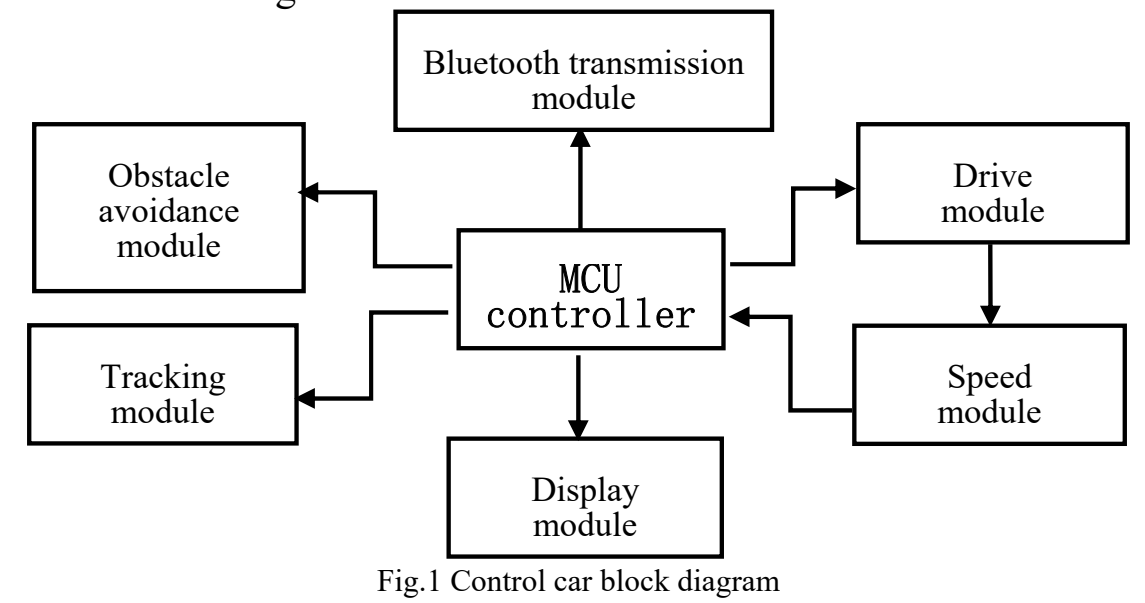

2.2 The hardware circuit design of intelligent car control system

\subsubsection{The selection of MCU}

This design uses the STC12C5A60S2 microcontroller. STC12C5A60S2 microcontroller is the basic content that is required to control the application of the integrated circuit chip [2]. Divide according to the function, which is composed of the following parts, the microprocessor, data memory, program memory, parallel I / O port, serial port, timer / counter, a PWM generator, a built-in watchdog, built-in 8-channel high-speed 10 bit a / D conversion, unique baud rate generator, interrupt system and special function registers. Each of them is connected to a single bus, and its basic structure is still the traditional structure mode of CPU plus peripheral chips.

2.2.2 The design of motor drive circuit

This design uses L293D chip, L293 is a high voltage, small current motor driver chip ST. The chip is packaged in a 16 pin, which is composed of an $\mathrm{H}$ bridge circuit which is composed of a bipolar tube. Its output current is $100 \mathrm{~mA}$, the maximum current is $2 \mathrm{~A}$, and the maximum working voltage is $36 \mathrm{~V}$, which can drive the inductive load, and can control the motor of the motor, and is easy to be controlled by single chip computer. Work with SCM control transistor in the switch state of adjustable duty ratio, precise adjustment of motor speed. Due to the saturation of the tube, the efficiency is very high. The $\mathrm{H}$ bridge circuit can be used to control the speed and direction of the speed and the stability is high.

2.2.3 The design of display circuit

LCD1602 character LCD screen is used in this design to display equipment, LCD display is widely used in many fields, such as portable computer, digital camera, PDA mobile communication tools and so on. DB0 DB7 - LCD port and the P0 port of the microcontroller, RS connected to the P2.7 port, RW P2.6 port, so that the port can be connected to the P2.5 port E. P0 port as data write end.

2.2.4 The design of Bluetooth module 
The Bluetooth module mainly realizes the data transmission between the upper computer and the lower computer. The design is the wireless communication between the upper computer and the lower computer through the Bluetooth. Using the WE-40C Bluetooth module, we set one for the host, and the other for the slave. To control the forward, backward and turn of the smart car by sending the AT command. WE-40C is easy to use, can support a pair of multi communication, but also through the AT command to switch the module's master slave mode. When the Bluetooth module is on the red indicator light flashes, and the other Bluetooth module is fast, the blue indicator light.

2.2.5 The design of speed measurement module

The function of the speed measurement module is to complete the current car speed information collection, and the output of the standard pulse signal, for single chip microcomputer processing, computing the real-time speed of the car. Speed detection can be achieved by the acceleration sensor, photoelectric encoding disk, and speed motor and so on. Adopt photoelectric infrared sensor. Uniform carved slot on the spoke panel, the wheels are fixed on both sides of the infrared transmitting and receiving device, relative[4].

\section{The software design of intelligent vehicle control system}

3.1 The design of main program

Figure 2 for the Bluetooth remote control car program flow chart, from the figure can be seen, the car can achieve Bluetooth remote control, and in the car driving process, automatic detection and control personnel. It can also automatically test the speed, the speed value sent to the host computer display.

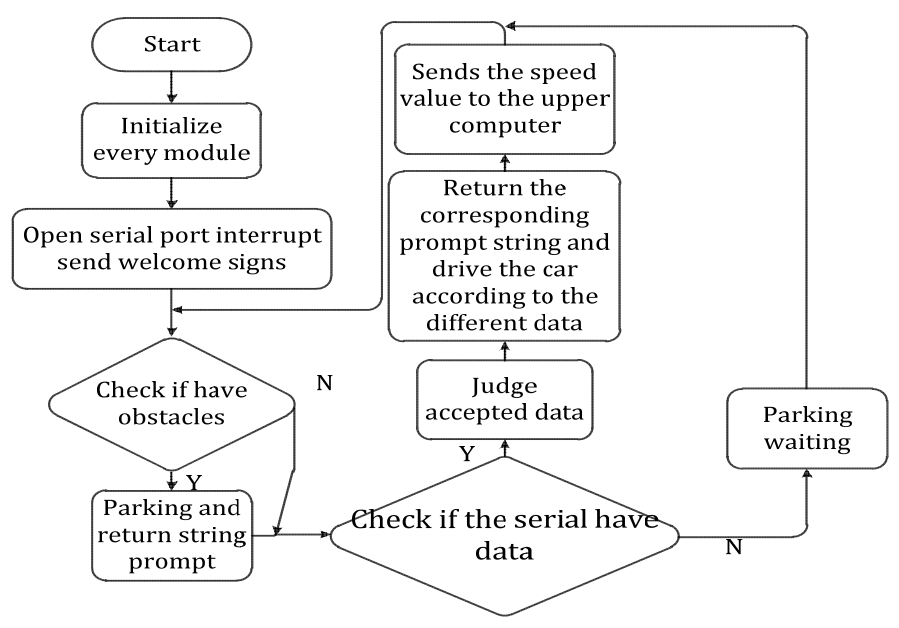

Fig. 2 Control car main program

In the main program is the most important part of the program is the serial communication program, microcontroller serial port communication is to achieve the host computer and the lower computer data transmission, SCM is through the RXD TXD and the external communication, SUBF is a serial port buffer register, the microcontroller and the communication data are stored in the SUBF register.

3.2 The design of ultrasonic obstacle avoidance program

The ultrasonic obstacle avoidance program is as Figure 3. 


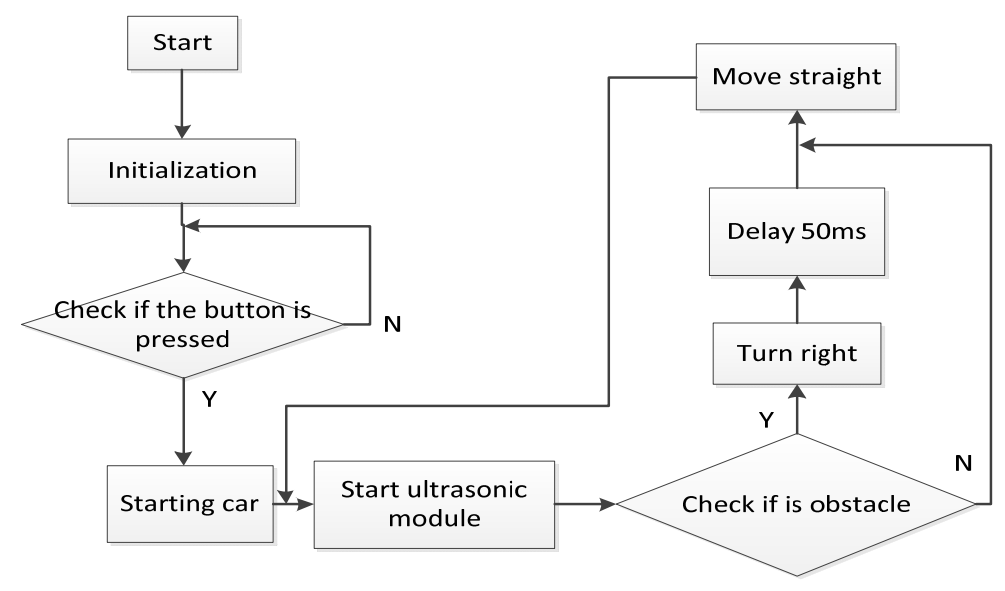

Fig. 3 Control car ultrasonic obstacle avoidance program

\section{Conclusion}

This design is to design the Bluetooth remote control multifunctional smart car based on experimental debugging to achieve the functions of smart car Bluetooth wireless remote control, automatic obstacle avoidance, automatic tracking and so on. In the design of the software using modular design ideas [6], the design of intelligent car operation is simple, stable and reliable, the system has broad potential and application value.

\section{References}

[1] He Li-min. Design of Bluetooth communication system based on Arduino/Android [M].Beijing: Aerospace University Press, 2002.

[2] LI Guang-di. Monolithic integrated circuit [M]. Beijing: University of Aeronautics and Astronautics Press, 2001.

[3] FU Qiang,REN Feng-hua. Design of remote control car based on mobile phone [J].Modern computer (Professional Edition), 2011, (31):77-79

[4] LIN Chang-qin,CHENG Hai-liang. Design of mobile remote control vehicle based on Android system and Bluetooth communication [J]. Shanxi Electronic Technology, 2012, (03):56-58

[5] Zheng Hao, ZHONG Zhifeng, GUO Hao. Design of Bluetooth communication system based on Arduino/Android [J]. Internet of Things Technologies, 2012, (5):50-51

[6] SUN Yu-yan. Data communication and control between PC and single chip microcomputer [J]. Journal of Guangzhou Baiyun Industrial and technical school, 2002, (04): 46-49 